\title{
Estudio del Contagio en Redes Financieras
}

\section{Study of the Contagion in Financial Networks}

Valeria L. Negrete Zambrano ${ }^{a}$, Pedro P. Romero Alemán ${ }^{b}$

\begin{abstract}
INFORMACIÓN DEL ARTÍCULO

Fecha de recepción: 18 de Septiembre de 2017.

Fecha de aceptación: 20 de Noviembre de 2017.

${ }^{a}$ Economista, Universidad San Francisco de Quito. Colaboradora del departamento de Economía de la Universidad San Francisco de Quito-Ecuador Email:valerianegrete94@hotmail.com.

${ }^{b} \mathrm{PhD}$ en Economía, George Mason University. Profesor del departamento de Economía de la Universidad San Francisco de Quito-Ecuador. Email: promero@usfq.edu.ec .
\end{abstract}

\begin{abstract}
Resumen
El objeto de este artículo fue estudiar la formación de redes financieras y determinar los shocks idiosincráticos frente a un contagio, a partir de la aplicación de los modelos de Gai y Kapadia, y Acemoglu, Ozdaglar y Tahbaz-Salehi. La teoría permitió la explicación del funcionamiento de la red y cómo se define la conexión de activos y pasivos interbancarios entre sí. Los estudios concluyeron que la cantidad de préstamos emitidos por un banco no debe sobrepasar los activos que este posee, ya que si llegara a suceder esto, el sistema estaría expuesto a una cascada de defaults. Además, que el contagio dependería del tamaño de grupos sensibles dentro de la red financiera, el grado de vulnerabilidad de cada banco, y cómo están conectados entre sí.
\end{abstract}

\section{Palabras Clave:}

Red financiera, contagio, riesgo sistemático, modelo de redes, crisis financiera.

\section{Clasificación JEL: D85, G21, G33}

\begin{abstract}
The purpose of this article was to study the formation of financial networks and determine idiosyncratic shocks in case of contagion, based on the application of Gai and Kapadia, and Acemoglu, Ozdaglar and Tahbaz-Salehi models. The theory explained the operation of the network and how the connection of interbank assets and liabilities is defined between them. The studies concluded that the amount of loans issued by a bank should not exceed the assets it owns, because if this happens, the system will be exposed to a cascade of defaults. In addition, contagion would depend on the size of sensitive groups within the financial network, the degree of vulnerability of each bank, and how they are connected to each other.
\end{abstract}

\section{Keywords}

Financial network, contagion, systematic risk, network models, financial crisis.

JEL Classification: D85, G21, G33

PODIUM No. 32, Diciembre 2017, pp. 31-43

Universidad Espíritu Santo - UEES

ISSN: 1390-5473 e-ISSN: 2588-0969 


\section{Introducción}

La reciente crisis financiera que sufrieron varios países, propagada alrededor del año 2008, ha dejado varias represalias en la economía mundial. Una de las principales causas fue la inestabilidad financiera de los principales bancos de inversión, compañías de seguros y empresas hipotecarias. El "Contagio en Redes Financieras", desarrollado por Gai y Kapadia (2009, 2010), junto a la contribución de Acemoglu, Ozdaglar y Tahbaz-Salehi (2014), son uno de los principales estudios para abordar el tema de este artículo, que implica estudiar la formación de redes financieras y determinar los shocks idiosincráticos frente a un contagio.

El objetivo principal consiste en analizar cómo las pérdidas de una institución financiera pueden ser propagadas a través de una red de exposiciones, mediante activos y pasivos interbancarios tras un default $^{1}$ inicial. La red bien puede ser extensa, es decir estar conectada con un sinnúmero de instituciones, o, pequeña, tan solo enlazada con pocos organismos.

Los sistemas financieros abarcan una red con enlaces de entradas y salidas que emiten préstamos y obligaciones. Estos están conectados por una variedad de intermediarios, es decir bancos y fondos de cobertura. Para representar las distintas razones de contagio se presentan dos opciones: 1) El modelo de Gai y Kapadia (2009, 2010), que es sencillo y propone que el incumplimiento puede extenderse de una institución a otra, y 2) El modelo de Acemoglu et al. (2014), que estudia la formación de redes financieras e investiga la interacción de los incentivos crediticios de los bancos y la aparición de riesgo sistemático.

Ahora bien, cuando la red está "completa", y todos los bancos están teniendo exposiciones entre sí, el impacto de un shock es fácilmente amortiguado. Por otra parte, cuando la red está "incompleta" y los bancos sólo tienen exposiciones frente a ciertas contrapartes, el sistema se vuelve más frágil, es decir, es más propenso a ser contagiado. El impacto inicial de un shock se concentra en los bancos adyacentes, una vez que estos perecen, la liquidación prematura de los activos a largo plazo y la pérdida de valor asociado, atraen bancos previamente no afectados frente al contagio.

La estructura arbitraria de la red y las interacciones entre los intermediarios financieros hacen que la dinámica del sistema no sea lineal. Por consiguiente, el riesgo de contagio puede ser muy sensible a pequeños cambios en los parámetros. Se analiza ésta característica dentro del modelo, aislando la probabilidad y propagación del contagio cuando los reclamos y las obligaciones están interrelacionados. Al hacerlo ofrece una perspectiva alterna sobre la cuestión de si el sistema financiero soporta el contagio o lo propaga extensamente. 


\section{Revisión de literatura}

Existe una extensa gama de autores que han explorado y analizado a profundidad sus contrapartes e implicaciones en la economía a nivel mundial. El estudio realizado por Gai y Kapadia (2009, 2010) determina que aunque la probabilidad de contagio sea baja, los efectos cuando se produzca pueden ser muy significativos. Sin embargo, Glasserman y Young (2016) estipulan que las conexiones de red pueden tener un efecto positivo al diversificar la exposición al riesgo de los bancos individuales.

Acemoglu et al. (2014) proponen un modelo que involucra el estudio de la formación de redes financieras e indaga la interacción entre los incentivos crediticios de los bancos y la aparición de riesgo sistemático. Mientras Chavarría (2014) analiza a los bancos más vulnerables y la presencia de cascadas, es decir, que un banco vaya a la quiebra por la caída de otros bancos, y así sucesivamente. Más allá de lo previsto, Allen y Gale (2000) mencionan que los mercados interbancarios completos son más robustos, valores que se ven afectados por pequeñas variaciones. En su publicación, Demange (2015) propone medir los efectos spill-over ${ }^{2}$ que generan los pasivos interbancarios sobre la propagación del impago, y estipula que las reclamaciones interbancarias son una preocupación para los reguladores.

Adicionalmente, Elliot, Golub, y Jackson (2014) explican cómo los cambios discontinuos en los valores de los activos provocan nuevos fallos, y cómo esto depende de la estructura de la red. De lo contrario, Leitner (2005), supone que en un tiempo igual a 0 , cada banco necesita tener un endeudamiento de al menos una unidad de bien para poder invertir; de lo contrario, sería imposible invertir. Nier, Yang, Yorulmazer, y Alentorn (2008) y Battiston, Gatti, Gallegatti, Greenwald, y Stiglitz (2009) concluyen en sus estudios, que el nivel de contagio y el rol de la interconexión dependen en gran medida de los detalles del modelo y de la estructura de la red. En el año 2000, Frexias, Parigi, y Rochet citaron que las conexiones interbancarias aumentan la resiliencia del sistema ante la insolvencia de un banco en particular. Por otro lado, se tiene a Amini, Cont, y Minca (2016), que tratan de medir cómo el contagio amplifica los pequeños shocks a la red. Sin embargo, Dasgupta y Kaligounder (2003) encontraron que mientras mayor sea el nivel de capitalización, más resistente se volverá el sistema al efecto de contagio, y por tanto, al efecto de grado de conectividad.

Otro propósito de examinar el contagio en redes lo presentan Caldarelli, Chessa, Pammolli, Gabrielli, y Puliga (2013), concluyendo que la extensión del contagio puede estar fuertemente entrelazado con la topología de la red de relaciones que une a los bancos y las instituciones financieras en el sistema. En 2008, May, Levin, y Sugihara, comentan la importancia de la estructura de interacción entre bancos e institucio-

$\overline{2}$ Efecto derrame, cuando el deudor no ha pagado la totalidad de las deudas que tiene con sus acreedores. 
nes financieras en la canalización y amplificación de los shocks que afectan a cualquier agente único en el sistema. Klinger y Teply (2014), confirman mediante un estudio de simulaciones que la suficiencia de capital en cada banco es crucial para la estabilidad del sistema, sin embargo, afirman que no es suficiente cuando el sistema ha entrado en colapso.

\section{Metodología}

Para analizar cómo las pérdidas de una institución financiera pueden ser propagadas a través de una red de exposiciones, mediante activos y pasivos interbancarios tras un default inicial, y representar las distintas razones de contagio se utilizaron los modelos de Gai y Kapadia $(2009,2010)$ y de Acemoglu et al. (2014).

\section{Estructura de red arbitraria}

Gai y Kapadia (2009) se enfocan en un análisis para definir la propagación de shocks de un banco aleatorio hacia otro y, así precisar los resultados analíticos que caracterizan un efecto cascada. $\mathrm{Se}$ considera una red financiera en la cual existen $n$ intermediarios financieros, es decir, bancos que están aleatoriamente unidos entre sí por sus préstamos. La figura 1 representa una red arbitraria directa en la cual hay cinco bancos conectados entre sí. Cada banco representa un nodo y los enlaces interbancarios definen los vínculos con otros bancos. La distribución de activos y pasivos interbancarios rige el potencial de propagación de un shock a través de la red, es decir, el tamaño de los enlaces interbancarios es importante para el análisis de contagio.

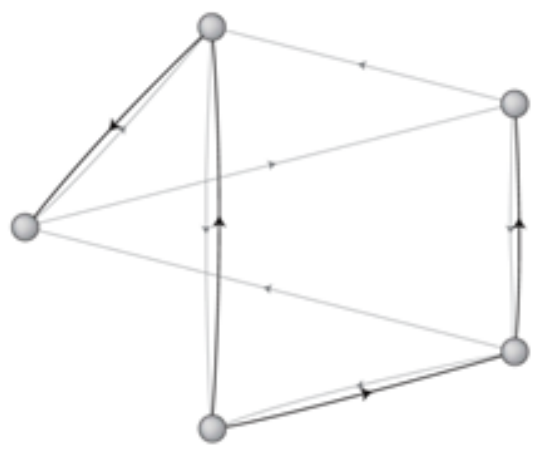

Figura 1. Red directa ponderada con 5 nodos. Fuente: Gai y Kapadia (2009)

Se define que $A_{i}^{I B}$ son activos interbancarios, mientras $A_{i}^{M}$, son hipotecas de los activos externos ilíquidos. Se asume que la posición total de los activos interbancarios de cada banco está distribuida equitativamente en cada uno de los enlaces que tiene el banco. Dicho esto, si un banco no tiene activos interbancarios, estos van a ser iguales a cero, $A_{i}^{I B}=0$. Se muestra que el contagio es posible cuando se maximiza la distribución del riesgo en el sistema.

Cada activo interbancario es el pasivo de otro banco, representado por $L_{i}^{I B}$. Se dice que una única unidad de pasivos interbancarios viene dado por los depósitos de los clientes $D_{i}$, siendo exógenos. La condición para que un banco i sea solvente, es la siguiente:

$$
(1-\phi) A_{i}^{I B}+q A_{i}^{M}-L_{i}^{I B}-D_{i}>0
$$

Se define que $\phi$ es la fracción de deuda, mientras que $q$ es el precio de reventa del activo ilíquido. El valor $q$, 
debe ser menor a 1 en caso de que exista venta de activos por parte de los bancos en incumplimiento, e igual a 1 si existen ventas de bienes con precios bajos debido a que el banco enfrenta una bancarrota.

Si un banco incumple con el pago y condiciones, el banco $i$ pierde todos sus activos interbancarios con dicha financiera. Por tanto, la condición de solvencia viene dada por la siguiente fórmula:

$$
\phi<\frac{\boldsymbol{K}_{i}-(\mathbf{1}-\boldsymbol{q}) \boldsymbol{A}_{i}^{M}}{A_{i}^{I B}}, \text { para } A_{i}^{I B} \neq \mathbf{0}
$$

Dicho esto se dice que todos los bancos de la red son solventes, en $t=1$. El fraude lleva al fracaso de las instituciones financieras. Por tanto, el fracaso bancario puede resultar de un shock agregado que tiene consecuencias adversas hacia una institución. Los enlaces entrantes para un banco $i$ es representado por $j$, mientras viene a $i$ ser la pérdida de los activos interbancarios cuando existe un incumplimiento, siempre y cuando esté dentro de los bancos vinculados. La condición cuando hay default viene dada por:

$$
\frac{K_{i}-(1-q) A_{i}^{M}}{A_{i}^{I B}}<\frac{1}{j_{i}}
$$

La probabilidad de que un banco sea vulnerable, depende de los enlaces de entrada $j$ y por tanto se define como:

$$
v_{j}=P\left[\frac{K_{i}-(1-q) A_{i}^{M}}{A_{i}^{I B}}<\frac{1}{j_{i}}\right] \forall j \geq 1
$$

Finalmente, se puede notar que para determinar las características del riesgo sistemático la estructura de la red viene definida desde las ecuaciones (3.1) hasta (3.4).

\section{Estructura endógena de red financiera}

Se demuestra que, si los shocks son por alguna razón extraños, los bancos no aceptan el hecho de que los préstamos pueden ayudar a nivelar el shock. A pesar de que los tipos de interés para cada prestatario se ajustan endógenamente estos reflejan el grado de riesgo de la contraparte.

Se considera una economía simple y consistente en $n$ bancos de riesgo neutral y financieros externos. En el modelo se prevé que la economía tiene una duración de tres periodos $\{t=0,1,2\}$. Durante la fecha inicial, cada banco i toma prestado fondos de otros bancos para invertir, lo cual producirá rendimientos en un periodo intermedio o final. Cada banco está dotado por k unidades de capital en $t=0$, que puede prestar al resto de entidades en toda la economía.

Se asume que $j$ representa un banco, este puede pedir prestado como máximo $k_{i j}$ unidades de capital del banco $i$. Es decir, $l_{i j} \leq k_{i j}$, donde $l_{i j}$ es la cantidad que el banco $i$ presta al banco $j$. Para toda entidad $i$ se asume que, $\sum_{j \neq i, 0} k_{i j} \geq k$, garantizando que todas las instituciones pueden recaudar fondos de otras entidades. Cada banco puede pedir prestado fondos a los financieros externos con un costo de oportunidad $r>1$, entre perio$\operatorname{dos} t=0$ y 1 . 
Préstamos interbancarios y contratos de deuda.

Los investigadores establecen que los préstamos interbancarios se realizan a través de contratos de deuda firmados en $t=0$, estos tienen que ser reembolsados en $t=1$. Las decisiones de endeudamiento de los bancos determinan la cantidad de préstamos interbancarios, es decir, $l_{i j}=b_{i j}$. Estos a su vez, condicionan la tasa de interés como $\widehat{R}_{i j}\left(l_{j 1}, \ldots, l_{j n}\right)$, lo que implica que el valor nominal de la deuda para el banco $j$ es igual a $y_{i j}=l_{i j} \widehat{R}_{i j}\left(l_{i 1}, \ldots, 1_{j n}\right)$.

Las denotaciones presentan características principales. La presencia de requisitos permite a los prestamistas cobrar diferentes tasas de interés, dependiendo la conducta de riesgo de sus prestatarios. En segundo lugar, a pesar de que la tasa de interés ofrecida por el banco $i$ a la entidad $j$ está condicionada al comportamiento crediticio de $j$, es por una parte independiente a los contratos $\boldsymbol{R}_{i}$ con sus otras contrapartes. Si los bancos $i$ y $j$ llegan a un acuerdo de préstamo, el préstamo sería igual a la capacidad de endeudamiento máximo.

\section{Inversión y reembolso de deuda}

Al momento de estar completos todos los préstamos interbancarios, el banco $i$ invertirá y obtendrá un reembolso al azar a corto plazo, como $z_{i} \in\{\mathrm{a}, \mathrm{a}-\epsilon\}$ en $t=1$, donde $\epsilon$, representa el tamaño de un shock negativo para el reembolso de la inversión, $a$. Si llegara a mantenerse hasta su vencimiento, la inversión tendrá una rentabilidad fija a largo plazo y no confiable en $t=2$. Por otro lado, el banco podrá liquidar su proyecto después del reembolso a corto plazo en $t=1$.

Una vez que el banco invierta, este debe cumplir con sus obligaciones $v>0$ en $t=1$. Por tanto, se puede decir que el total de deudas del banco $i$ en $t=1$ es igual a $\mathrm{y}_{i}+1$ donde $\mathrm{y}_{i}=\sum_{j \neq i} \mathrm{y}_{j i}$. Ahora bien, si el banco $j$ no puede cumplir con sus obligaciones, debe liquidar la inversión cuando los ingresos se distribuyan entre sus otros acreedores. Consecuentemente, si el banco $j$ puede cumplir con sus obligaciones $v$, pero incumpliendo su deuda con los otros acreedores, ellos deben pagar en proporción al valor nominal de los contratos. Y, si no pueden cumplir su obligación externa, sus acreedores no reciben nada.

\section{Equilibrio de pago}

Se considera un subjuego en el cual el valor nominal de las obligaciones interbancarias vienen dadas por $\left\{y_{j i}\right\}$, y el banco $\mathrm{j}$ mantiene una cantidad de su exceso de capital como efectivo, $c_{j}=k-\sum_{j \neq i} l_{j i}$. Se considera que, $x_{j s}$ es el reembolso por el banco $s$ de su deuda al banco $j$ en $t=1$. Se dice también que $h_{j}=\mathrm{c}_{j}+\mathrm{z}_{j}+\sum_{s \neq j} x_{j s}$, representa el flujo de caja total.

Si $h_{j}$ es mayor que el total de los pasivos de un banco, $v+y_{j}$, implica que el banco puede cumplir con sus obligaciones en su totalidad. Esto beneficia a los acreedores ya que reciben un pago igual al valor nominal de sus reclamos. Es decir, $x_{i j}=y_{i j}$ para todo $i \neq j$. Por el otro lado, si $\mathrm{h}_{j}>v+\mathrm{y}_{j}$, indica que el banco $\mathrm{j}$ incumple $\mathrm{y}$ sus prestadores pagan menos que su valor nominal. Es decir, el banco no 
cumple con sus obligaciones y sus acreedores junior no reciben nada. Por tanto, $x_{i j}=0$.

Ahora bien, si $h_{j} \in\left(v, v+y_{j}\right)$, los pagos de la deuda por el banco $j$ a sus acreedores junior son proporcionales al valor nominal de los contratos. Para resumir, el pago $t=1$ del banco $j$ a un banco acreedor $i$, se lo simboliza:

$$
x_{i j}=\frac{y_{i j}}{y_{j}}\left[\min \left\{y_{j}, c_{j}+z_{j}-v+\sum_{s \neq j} x_{j s}\right\}\right]^{+}
$$

donde $[\cdot],{ }^{+}$es representado por max $\{\cdot, 0\}$. Si el banco no puede cumplir con sus obligaciones, este tiene que liquidar su plan prematuramente. Sin embargo, el equilibrio se puede definir en $t=1 \mathrm{del}$ juego como el conjunto interbancario mutuamente consistentes.

\section{Resultados}

Se presentarán los resultados matemáticos ofrecidos por Gai y Kapadia (2009, 2010) y Acemoglu et al. (2014) en sus investigaciones como tal.

\section{Estructura de red arbitraria: Generación de funciones y la transmisión de shocks}

Dentro de una red financiera, si el número de nodos es suficientemente grande, existe muy poca probabilidad que los intermediarios estén expuestos a más instituciones con incumplimientos después de haber sufrido un contagio. Estableciendo que una entidad segura nunca fallará en un segundo contagio.
Por tanto, la probabilidad de la función de generación es simplemente la siguiente:

$$
\mathcal{G}(x, y)=\sum_{j, k} v_{j} \cdot p_{j k} \cdot x^{j} \cdot y^{k}
$$

donde $p_{j k}$ es el grado de distribución y $v_{j}$ es la distribución de vulnerabilidad.

Se debe agregar que cada enlace de salida para un nodo es un enlace de entrada para otro, y para cada activo interbancario de un banco es un pasivo interbancario de otro. El promedio de enlaces de entrada de una red debe ser igual al promedio de enlaces de salida. Es decir:

$$
\frac{1}{n} \sum_{i}^{j_{i}}=\sum_{j, k} j p_{j k} \rightarrow \frac{1}{n} \sum_{i} k_{i}=\sum_{j, k} k p_{j k}
$$

donde, $\mathrm{G}(\mathrm{x}, \mathrm{y})$ define la función de generación, mientras que G_0 (y) representa el la fracción de bancos vulnerables. Lo cual es denotado implícitamente por:

$$
\mathcal{G}(1,1)=G_{0}(1)=\sum_{j, k} v_{j} \cdot p_{j k}
$$

Bajo este preámbulo, se toma en cuenta los grados de distribución de bancos vulnerables al azar, representados por $v_{j} \cdot r_{j k}$.

Se nota que la función de generación, $G_{1}(y)$, por el número de enlaces de salida de un banco aleatorio vulnerable, viene dado por:

$$
G_{1}(y)=\sum_{j, k} v_{j} \cdot r_{j k} \cdot y^{k}=\frac{\sum_{j, k} v_{j} \cdot j \cdot p_{j k} \cdot y^{k}}{\sum_{j} j \cdot p_{j k}}
$$


Se dice que, $H_{1}(y)$, es la función de generación para la probabilidad de llegar a un grupo de bancos vulnerables que captan enlaces de salida. Este satisface la siguiente condición de autoconsistencia dentro de la red:

$H_{1}(y)=\operatorname{Pr}[$ reach safe bank $]+y \sum_{j, k} v_{j} \cdot r_{j k} \cdot\left[H_{1}(y)\right]^{k}$

donde y es una variable discreta aleatoria que toma valores de $\{0,1,2,3 \ldots\}$, mientras $\operatorname{Pr}=1$.

Usando la ecuación (4.3) y la notación $\mathrm{G}_{1}(1)$, representa la probabilidad de que un banco adyacente aleatorio sea vulnerable. Teniendo la ecuación (4.4), se asume la notación consiguiente:

$$
\begin{gathered}
G_{1}(y)=\sum_{j, k} v_{j} \cdot r_{j k} \cdot y^{k}=\frac{\sum_{j, k} v_{j} \cdot j \cdot p_{j k} \cdot y^{k}}{\sum_{j} j \cdot p_{j k}} \\
G_{1}(1)=\sum_{j, k} v_{j} \cdot y_{j k} \cdot y^{k}-\sum_{j . k j} v_{j} \cdot y^{k} \\
G_{1}(1)=y G_{1}(1) \cdot\left[H_{1}(y)\right]^{k} \\
H_{1}(y)=\operatorname{Pr}[\text { reach safe bank }]+y \sum_{j, k} y_{j} \cdot r_{j k} \cdot\left[H_{1}(y)\right]^{k}-G_{1}(1) \\
\boldsymbol{H}_{\mathbf{1}}(\boldsymbol{y})=\mathbf{1}-\boldsymbol{G}_{\mathbf{1}}(\mathbf{1})+\boldsymbol{y} \boldsymbol{G}_{\mathbf{1}}\left(\boldsymbol{H}_{\mathbf{1}}(\boldsymbol{y})\right)
\end{gathered}
$$

Dicho lo anterior, ahora se establece la distribución de los tamaños de grupos sensibles que capten enlaces de salida. Pueden existir dos posibles soluciones; primero, un banco elegido al azar puede ser seguro. Puede tener enlaces de salida y entrada, y ser vulnerables con probabilidad $\mathrm{v}_{j} \cdot \mathrm{p}_{j k}$, sin embargo cuando cada enlace de salida conduce a un grupo frágil al que pertenece un banco al azar, es generado por la siguiente fórmula:

$$
H_{0}(y)=1-G_{0}(1)+y G_{0}\left(H_{1}(y)\right)
$$

\section{Probabilidad y propagación de contagio.}

La probabilidad de contagio depende del tamaño de grupos sensibles dentro de la red. En la transición de fases, la posibilidad de que exista un riesgo debe ser cercana a cero, mientras que el tamaño del conjunto vulnerable es restringido por una alta fracción de bancos seguros. Los efectos de dispersión del riesgo de un sistema más enlazado, aumentará el tamaño del grupo frágil y la probabilidad de contagio. Un banco seguro puede ser susceptible al default y el contagio se puede propagar más para afectar a toda la red conectada.

\section{Estructura endógena de red financiera}

Acemoglu et al. (2014) presentan un modelo en el cual muestran la aparición de riesgo sistemático, y afirman que las redes financieras de equilibrio pueden ser excesivamente propensas al riesgo de contagio financiero.

\section{Eficiencia Bilateral}

Se considera una economía por tres bancos $\{1,2,3\}$, cada uno dotado por $k$ unidades de capital. Para que puedan invertir cada uno en sus proyectos respectivos, los bancos 1 y 2 necesitan pedir prestado $k$ de las entidades 2 y 3 . El banco 3 no toma prestado y solamente actúa como prestamista para la entidad 2. Por 
otro lado, si ningún banco pide ayuda a los financieros externos para obtener fondos, se formará una cadena de 3 redes, como se muestra en la figura 2:

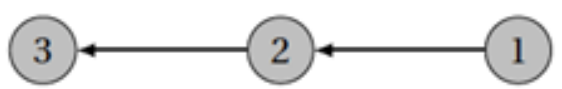

Figura 2. Cadena de 3 redes financieras. Fuente: Acemoglu et al (2014)

Se asume que el banco 1 es la única entidad sujeta a un shock negativo, y si dentro de la cadena de tres redes éste emite un shock negativo, conllevará el fracaso de todos los bancos. Sin embargo, los bancos 2 y 3 no están propensos a shocks. Se considera que $z_{1} \in\{a-\epsilon, a\}$, donde el shock negativo se realiza con probabilidad $p$ y satisface $2(a-v)<\epsilon<$ $2(a-v)+k$.

Ahora bien, los beneficios del banco 3 dependen si la entidad 2 decide prestar al banco 1. Un acuerdo de préstamo bilateral entre los bancos 1 y 2 hace aumentar la probabilidad de incumplimiento del banco $2 \mathrm{y}$, al mismo tiempo expone a la entidad 3 al riesgo de contagio.

Proposición 1: la cadena de tres redes financieras forma parte de un equilibrio sólo si es eficiente. De igual modo, es eficiente sí solo si cumple la siguiente condición:

$$
(r-1) k \geq p A
$$

Puesto que solo hay dos posibles contratos bilaterales interbancarios, sólo pueden surgir cuatro posibles redes financieras. Si los bancos 2 y 3 prestan a 1 y 2 , el excedente social satisface a:

$$
u_{3-\text { cadena }}=2_{a}-p \epsilon+k+2(1-p) A
$$

donde 2(1-p)A representa el hecho de que el banco 1 lleva al default de las entidades 1 y 2 .

\section{Externalización de la red financiera: exceso de préstamo}

Los investigadores consideran una economía en la que el banco $i$ no puede tomar prestado de cualquier banco que no sea el banco $i+1$, mientas que la entidad $n$ sólo puede pedir prestado a la financiera 1. Por tanto, si todas las inversiones son financiadas por préstamos interbancarios, la red financiera se formaría como anillo, representado en la figura 3 .

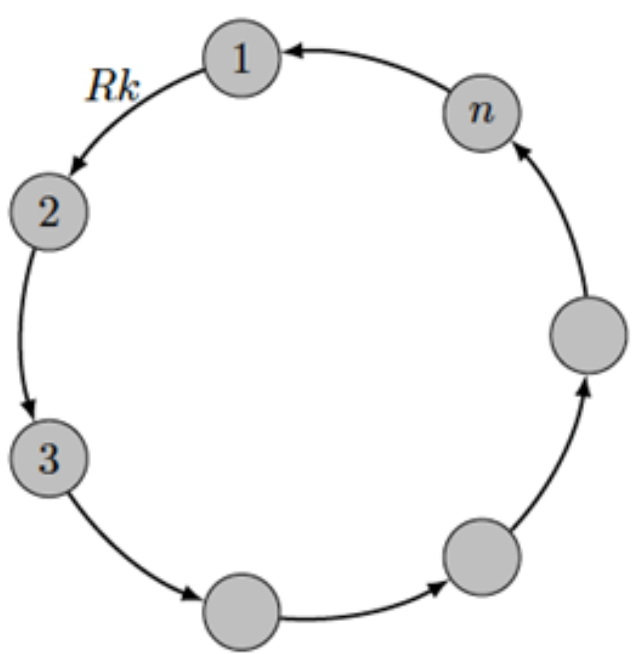

Figura 3. Red financiera de anillo Fuente: Acemoglu et al (2014) 
Proposición 2: un banco es afectado por un shock $\epsilon<n(a-v)$. Sin embargo, implican constantes que son condicionas de la siguiente manera:

a) Red financiera de anillo es ineficiente si $(r-1) k<\bar{\alpha} A$

Será socialmente ineficiente siempre y cuando los costos asociados con el mayor riesgo de contagio financiero sean altos. La red financiera de anillo es simétrica, hay $\tau$ incumplimientos en un solo shock negativo.

Los eruditos muestran que la presencia de externalidades en la red financiera implica que la estabilidad financiera es un bien público que es sobreprestamista en equilibrio.

\section{Externalidad de la red financiera: sub-diversificación}

Los investigadores presentan una posible sub-diversificación de la red, se centran en una economía en la cual cada banco puede prestar a dos prestatarios diferentes. Si bien consideran una economía $n$-bancaria, donde $n$ representa un número par. Donde cada banco $2 i$ y $2 i-1$ pueden prestar a los bancos $2 i-2$ y $2 i-3$. Se representa a una red financiera de anillos interconectados cuando, todos los bancos deciden prestar por igual a sus prestatarios (ver figura 4):

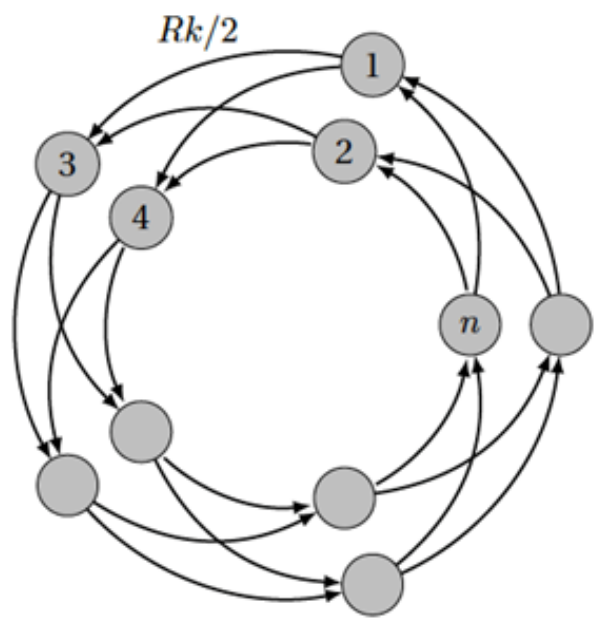

Figura 4. Red financiera de anillos interconectados Fuente: Acemoglu et al (2014)

Cada banco puede tomar una estrategia de préstamo menos diversificada, esto conduce a la formación de una red financiera de doble anillo (ver figura 5).

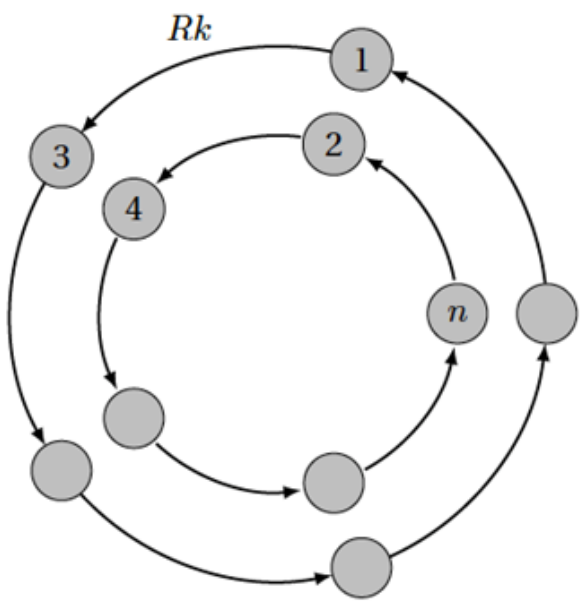

Figura 5. Red financiera de doble anillo Fuente: Acemoglu et al (2014) 
Proposición 3: un banco es afectado por un shock al azar, entonces $\epsilon<\frac{n(a-v)}{2}$, donde existe $\alpha>0$.

a) Red financiera de doble anillo socialmente ineficiente.

El contagio financiero se reduce cuando los bancos distribuyen sus préstamos entre más contrapartes. Si se reduce el efecto cascada, una estrategia beneficiará a los bancos que estén más arriba de la cadena de crédito.

\section{Crisis Sistemática}

La crisis es importante a nivel de bienestar social, es sustancial enfocarse en los préstamos interbancarios. Donde cada banco $i$ puede pedir prestado a cualquier otra entidad $j$ un aporte mínimo de $k_{j i}=k /(n-1)$. Mientras, si ningún banco decide acumular efectivo, aparecerá una red financiera completa, como se observa en la figura 6 .

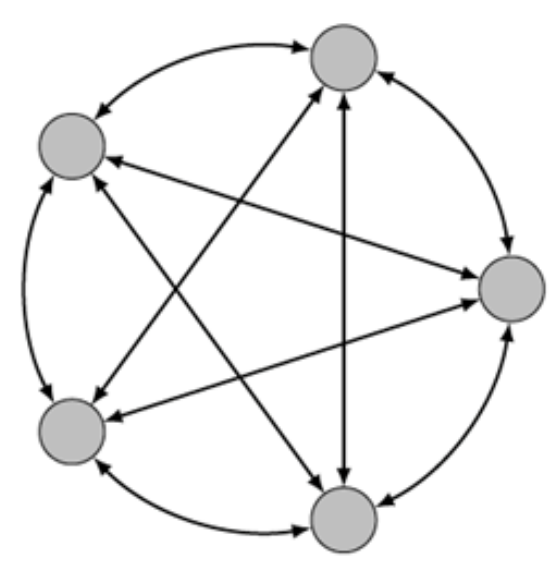

Figura 6. Red financiera completa Fuente: Acemoglu et al (2014)
Proposición 4: red financiera completa, $\epsilon^{*}=n(a-v)$, y que un solo banco es afectado por un shock negativo.

a) Si $\epsilon<\epsilon^{*}$, la red completa tiene un mínimo de defaults donde sólo un banco falla.

Si el tamaño del shock es pequeño, la red completa es la red más estable en la que ningún banco tiene defaults. Puesto que, dentro de la red completa los defaults de bancos en dificultades se van a dividir entre muchos acreedores más, garantizando que el exceso de liquidez en el sistema financiero puede absorber los defaults.

b) Si $\epsilon>\epsilon^{*}$, la red completa tiene un máximo de defaults donde todos los bancos fallan

Frente a todos los shocks, la red completa es la más frágil de todas las redes financieras, donde las cascadas conducen al fracaso de todos los bancos.

\section{Discusión}

Se evidencia que para comprobar un contagio en redes financieras es necesaria la realización de estudios empíricos, y gracias a los sistemas de iteración de Monte Carlo y MATLAB se pueden verificar los shocks negativos existentes en los bancos y cómo se propaga la crisis. Los datos más importantes que se requieren para el estudio son: depósitos a terceros, inversiones, créditos a otros bancos, deuda con el Banco Central y patrimonio. 
Otro punto de discusión es el tamaño de la red. Si la red es suficientemente grande, existe muy poca probabilidad que los intermediarios estén expuestos a más instituciones con incumplimientos después de haber sufrido un segundo contagio. Si un banco aleatorio conectado a la red es vulnerable, y si el valor de los enlaces promedio de pasivos son menores a 1, el contagio desaparecerá, debido a que el número de instituciones vulnerables disminuye. Caso contrario, en una red grande con pasivos mayores a 1, el contagio se puede propagar a través de toda la red financiera, debido al incumplimiento de pago.

Finalmente, gracias a los resultados alcanzados con las debidas simulaciones y con una correcta interpretación se pudo comprobar cuál es el indicador de vulnerabilidad de un banco y determinar las relaciones de deuda de diferentes contrapartes.

\section{Conclusiones}

Para determinar el contagio se realizó un estudio analítico con el fin de establecer cómo está estructurada la red. El primer modelo establecido presentó una estructura arbitraria donde el número de enlaces determinó cuan propensos son a esparcir un contagio. De la misma manera se comprobó que el fraude conduce al fracaso de las instituciones. Para que no exista riesgo sistemático, las entidades financieras deben ser vulnerables, esto depende tanto de los enlaces de entrada y salida. Sin embargo, si una institución posee un mayor número de enlaces entrantes, este reduciría el riesgo de com- partir los beneficios de mayor conectividad. Aunque la vulnerabilidad de un banco disminuya de una manera lenta, la probabilidad de contagio seguirá siendo considerablemente alta. Por otro lado, si la posición total de activos interbancarios crece, el grado de vulnerabilidad aumentará sin tergiversación el riesgo de contagio.

Por último, si el número de nodos es grande, la probabilidad de que los intermediarios estén expuestos a más instituciones con incumplimientos después de haber sufrido un contagio, es muy baja. De igual manera se comprueba que un banco seguro nunca fallará en un segundo contagio, por ende, la probabilidad de contagio está relacionada con el tamaño de grupos sensibles dentro de la red financiera. Esto implica que el contagio depende tanto del tamaño, grado de vulnerabilidad de cada banco y cómo están conectados entre sí.

\section{Referencias}

Acemoglu, D., Ozdaglar, A., y Tahbaz-Salehi, A. (2014). Systemic Risk in Endogenous Financial Networks. Kellog School of Management, Northwestern University.

Allen, F. y Gale, D. (2000). Financial contagion. Journal of Political Economy 108, 1-33.

Amini, H., Cont, R., y Minca, A. (2016). Resilience to Contagion in Financial Networks. Mathematical Finance: An International Journal of Mathematics, Statics and Financial Economics, 26(2), 329-365. 
Battiston, S., Gatti, D., Gallegatti, M., Greenwald, B., y Stiglitz, J. (2009). Liaisons dangerousness: increasing connectivity, risk sharing, and systemic risk. Journal of Economic Dynamics and Control, 36 (8), 1121-1141.

Caldarelli, G., Chessa, A., Pammolli, F., Gabrielli, A., y Puliga, M. (2013). Reconstructing a credit network, Nature Physics, 9, 125-126.

Chavarría, A. (2014). Redes Bancarias y Riesgo Sistemático: Desarrollo de un Algoritmo de Análisis y Diagnóstico. Centro de Regulación y Estabilidad Macrofinanciera. Facultad Economía y Negocios, Universidad de Chile.

Dasgupta, B. y Kaligounder, L. (2003). On global stability of financial networks. Journal of Complex Networks, 2(3), 313-354.

Demange, G. (2015). Contagion in Financial Networks: A Threat Index. CESifo, Center for Economic Studies \& Ifo Institute. Working Paper No. 5307.

Elliot, M., Golub, B., y Jackson, M. (2014). Financial Networks and Contagion. American Economic Review, 104(10), 3115-3153.

Freixas, X., Parigi, B., y Rochet, J. (2000). Systemic risk, interbank relations, and liquidity provision by the central bank. Journal of Money, Credit and Banking, 32, 611-638.

Gai, P. y Kapadia, S. (2009). Contagion in financial networks. Crawford School of Economics and Government. Bank of England.

Gai, P. y Kapadia, S. (2010). Contagion in financial networks. Bank of England. Working Paper No. 383.

Glasserman, P. y Young, P. (2016). Contagion in Financial Networks. Journal of Economic Literature,54(3), 779-831.

Klinger, T. y Teply, P. (2014). Systemic Risk of the Global Banking System an Agent-Based Network Model Approach. Prague Economic Papers, 1. Leitner, Y. (2005). Financial Networks: Contagion, Commitment, and Private Sector Bailouts. The Journal of Finance,60(6).

May. R, Levin, S., y Sugihara, G. (2008). Ecology for bankers. Nature, 451, 893-895.

Nier, E., Yang, J., Yorulmazer, T., y Alentorn, A. (2008). Network Models and Financial Stability. Bank of England. Working Paper No. 346. 